\title{
3D Short-Range Wetting and Nonlocality
}

\begin{abstract}
A. O. Parry, ${ }^{1}$ C. Rascón, ${ }^{2}$ N. R. Bernardino, ${ }^{1}$ and J. M. Romero-Enrique ${ }^{3}$
${ }^{1}$ Department of Mathematics, Imperial College London, London SW7 2BZ, United Kingdom

${ }^{2}$ Departamento de Matemáticas, Universidad Carlos III de Madrid, 28911 Leganés, Spain

${ }^{3}$ Departamento de Física Atómica, Molecular y Nuclear, Universidad de Sevilla, 41080 Seville, Spain

(Received 26 November 2007; revised manuscript received 21 January 2008; published 4 April 2008)

Analysis of a microscopic Landau-Ginzburg-Wilson model of 3D short-ranged wetting shows that correlation functions are characterized by two length scales, not one, as previously thought. This has a simple diagrammatic explanation using a nonlocal interfacial Hamiltonian and yields a thermodynamically consistent theory of wetting in keeping with exact sum rules. For critical wetting the second length serves to lower the cutoff in the spectrum of interfacial fluctuations determining the repulsion from the wall. We show how this corrects previous renormalization group predictions for fluctuation effects, based on local interfacial Hamiltonians. In particular, lowering the cutoff leads to a substantial reduction in the effective value of the wetting parameter and prevents the transition being driven first order. Quantitative comparison with Ising model simulation studies due to Binder, Landau, and co-workers is also made.
\end{abstract}

DOI: 10.1103/PhysRevLett.100.136105

PACS numbers: 68.08.Bc, 05.70.Fh, 05.70.Np

Progress has recently been made towards resolving problems in the theory of three-dimensional (3D) shortranged wetting [1-3] from analysis of a nonlocal (NL) interfacial Hamiltonian [4-6]. Starting from a LandauGinzburg-Wilson (LGW) model, it can be shown that the interfacial binding potential contains two-body interfacial interactions and also has a simple diagrammatic representation $[5,6]$. The latter allows one to visualize the binding potential as arising from tubelike fluctuations that zigzag between the surfaces. Numerical and renormalization group (RG) studies of critical wetting using the NL Hamiltonian [4] are in better agreement with Monte Carlo simulations of the transition in the 3D Ising model $[2,3]$. As is well known, the latter reveal neither the strong nonuniversal criticality [1] nor the stiffness-instability behavior [7] predicted using local interfacial Hamiltonians.

In this Letter, we highlight the specific mechanism by which nonlocality resolves long-standing problems in the theory of 3D wetting. From reanalysis of a LGW model, we show that correlation functions are characterized by, not one but, two diverging parallel length scales. Similar to the binding potential, this also has a simple diagrammatic explanation within the NL theory and shows how correlations arise from the interaction of interfacial and tubelike fluctuations. The appearance of a second diverging length has important consequences: First, the NL model is thermodynamically consistent, satisfying an exact correlation function sum rule due to Henderson [8]. Second, for critical wetting the second length lowers the cutoff of interfacial fluctuations controlling the repulsion from the wall. This simple mechanism pinpoints some inadequacies of previous effective Hamiltonian studies [1,7]. In particular, it prevents a stiffness instability [7], preserving the continuous nature of the transition, and leads to a lower, effective value for the wetting parameter controlling the nonuniversality implying that critical singularities are much closer to their mean-field predictions. This allows positive comparison with the results of previously problematic Ising model simulations $[2,3]$.

Imagine bringing a planar wall into contact with a bulk phase $\alpha$. In general, a microscopic layer of the preferred phase $\beta$ will intrude between the wall and the $\alpha$ phase. At a wetting transition [9-11], the thickness of this layer $\ell$ diverges. This situation occurs on approaching two-phase coexistence and the wetting temperature $T_{w}$, and may be first order or continuous (critical wetting). Alternatively, the divergence of $\ell$ on approaching two-phase coexistence for $T>T_{w}$ is termed complete wetting. As the film thickens, a fluid interface between phases $\alpha$ and $\beta$ is subject to increasingly large fluctuations characterized by a parallel correlation length $\xi_{\|}$. For critical and complete wetting, both $\ell$ and $\xi_{\|}$diverge continuously and are described by critical exponents [11]. For systems with short-ranged forces, the upper critical dimension for both transitions is $d=3$, and RG studies [1] of a standard (local) interfacial Hamiltonian predict that critical wetting has strongly nonuniversal critical singularities determined by the value of the wetting parameter

$$
\omega=k_{B} T / 4 \pi \Sigma \xi^{2}
$$

where $\Sigma$ is the interfacial stiffness and $\xi \equiv 1 / \kappa$ is the correlation length of the bulk $\beta$ phase. For example, on approaching $T_{w}$ at bulk coexistence, $\xi_{\|} \sim\left(T_{w}-T\right)^{-\nu_{\|}}$, where $\nu_{\|}(\omega)=(\sqrt{2}-\sqrt{\omega})^{-2}$ for $1 / 2<\omega<2$.

The ideal testing ground for this prediction is the Ising model for which $\omega \approx 0.8$ close to $T_{c}$ [12] suggesting $\nu_{\|} \approx$ 3.7, much larger than the mean-field (MF) value $\nu_{\|}=1$. In sharp contrast to this, extensive simulation studies by Binder et al. [2] found only minor deviations from MF theory, attributable to a much smaller effective value $\omega_{\text {eff }} \approx 0.27 \pm 0.12$, lying somewhere between $\mathrm{MF}$ and 
RG expectations [3]. The situation was confounded by a further refinement of the model, incorporating a positiondependent stiffness, due to Fisher and Jin [7] who predicted that, paradoxically, fluctuations drive the transition first order, in disagreement with the qualitative findings of the simulations.

To continue, we return to the starting point of wetting theory and, using a magnetic notation, consider a LGW model $[9,10]$ based on a magnetizationlike order parameter $m(\mathbf{r})$

$$
\mathcal{H}[m]=\int d \mathbf{r}\left\{\frac{1}{2}(\nabla m)^{2}+\phi(m)\right\},
$$

which has a bounding wall in the $z=0$ plane. A doublewell potential $\phi(m)$ describes the coexistence of bulk phases $\alpha$ and $\beta$ where, in zero field, $m_{\beta}=-m_{\alpha}=m_{0}$, where $m_{0}$ is the spontaneous magnetization. The bulk ordering field satisfies $h \leq 0$ so the bulk phase is $\alpha$. Minimization of (2) determines the MF equilibrium profile $\langle m\rangle=m(z)$. With a fixed wall magnetization $m=m_{1}>0$, the model exhibits critical wetting when $m_{1}=m_{0}\left(T_{w}\right)$.

Next consider the correlation function $G\left(\mathbf{r}_{1}, \mathbf{r}_{2}\right)=$ $\left\langle m\left(\mathbf{r}_{1}\right) m\left(\mathbf{r}_{2}\right)\right\rangle-\left\langle m\left(\mathbf{r}_{1}\right)\right\rangle\left\langle m\left(\mathbf{r}_{2}\right)\right\rangle$ and its parallel Fourier transform (FT) $\mathcal{G}$, which, at MF level, satisfies

$$
\left[-\partial_{z_{1}}^{2}+\phi^{\prime \prime}\left(m\left(z_{1}\right)\right)+q^{2}\right] \mathcal{G}\left(z_{1}, z_{2} ; q\right)=\delta\left(z_{1}-z_{2}\right),
$$

where we have set $k_{B} T=1$. This can be solved within the double-parabola (DP) approximation $\phi(m)=\kappa^{2}(|m|-$ $\left.m_{0}\right)^{2} / 2-h m$, which is known to describe the physics of continuous wetting transitions [6]. Within the DP approximation, the MF thickness [defined by $m(\ell)=0$ ] is $\kappa \ell \approx$ $\ln \left[-(t / 2)+\sqrt{\left(t^{2} / 4\right)-\tilde{h}}\right]$ where $t \equiv m_{1} / m_{0}-1$ and $\tilde{h} \equiv$ $h /\left(m_{0} \kappa^{2}\right)$. This displays the well-known logarithmic divergences of $\ell$ at critical and complete wetting. The result for $G=G^{\text {sing }}+G^{\text {reg }}$ separates conveniently into singular and regular parts. The regular part contains no diverging length scales while the singular term is found by calculation to be [13]

$$
\mathcal{G}^{\text {sing }}\left(z_{1}, z_{2} ; q\right)=\frac{\Psi\left(z_{1} ; q\right) \Psi\left(z_{2} ; q\right)}{E(\ell ; q)},
$$

where, in the relevant long wavelength limit $(q \ll \kappa)$, $\Psi(z, q) \approx m_{0} \kappa e^{-\kappa_{q}(\ell-z)}$, and

$$
E(\ell ; q) \approx 2 m_{0} \kappa|h|+2 m_{0}^{2} \kappa^{3} e^{-2 \kappa \ell} e^{-q^{2} \ell / \kappa}+\sigma_{\alpha \beta} q^{2} .
$$

Here, $\kappa_{q}=\sqrt{\kappa^{2}+q^{2}}$ and $\sigma_{\alpha \beta}=m_{0}^{2} \kappa$ are the DP results for the interfacial tension or stiffness. One finds from (4) that the correlation function is determined by two diverging parallel length scales: the usual parallel correlation length $\xi_{\|}=\sqrt{\sigma_{\alpha \beta} / E(\ell ; 0)}$ and also $\xi_{\mathrm{NL}}=\sqrt{\ell \bar{\xi}} \propto \sqrt{\ln \xi_{\|}}$. For example, in the approach to critical wetting at coexistence, and near the interface

$$
G(\ell, \ell ; q) \approx \frac{G(\ell, \ell ; 0)}{e^{-q^{2} \xi_{\mathrm{NL}}^{2}}+q^{2} \xi_{\|}^{2}},
$$

where $G(\ell, \ell ; 0)=\kappa^{2} m_{0}^{2} \xi_{\|}^{2} / \sigma_{\alpha \beta}$, while near the wall

$$
\mathcal{G}^{\operatorname{sing}}(0,0 ; q) \approx \frac{1}{2 \kappa} \frac{e^{-q^{2} \xi_{\mathrm{NL}}^{2}}}{e^{-q^{2} \xi_{\mathrm{NL}}^{2}}+q^{2} \xi_{\|}^{2}} .
$$

The wave-vector dependence of these correlation functions, characterized by two diverging parallel length scales, corresponds to a breakdown of simple scaling and is rather revealing.

Before we discuss this, note that all the above is captured by the NL model, which describes wetting for both planar and nonplanar walls, and may be derived from the LGW model [5]. A collective coordinate $\ell(\mathbf{x})$ denotes the location of a surface of isomagnetization $m\left(\mathbf{r}_{\ell}\right)=0$ where $\mathbf{r}_{\ell}=(\mathbf{x}, \ell)$ is an arbitrary point on the interface. Let $\psi(\mathbf{x})$ describe the shape of the substrate. A trace over irrelevant fluctuations identifies $H[\ell]=\mathcal{H}\left[m_{\Xi}\right]$ [7] where $m_{\Xi}(\mathbf{r})$ is the profile that minimizes (2) subject to the boundary conditions. For the DP potential, one finds

$$
\begin{aligned}
& m_{\Xi}(\mathrm{r})-m_{\beta}=-m_{\beta}(\underline{\boldsymbol{j}}-\underline{\mathfrak{L}}+\cdots) \\
& +\left(m_{1}-m_{\beta}\right)(22-25+\cdots)
\end{aligned}
$$

where the thick straight line denotes Green's function $K(\mathbf{r})=\kappa e^{-\kappa r} / 2 \pi r$ and the wavy lines represent the interfacial configuration (top) and wall (bottom). A black dot on a surface means one must integrate over all points on that surface with the appropriate infinitesimal area element (Fig. 1). The NL Hamiltonian is

$$
H[\ell]=\sigma_{\alpha \beta} A_{\alpha \beta}+2 m_{0}|h| V_{\beta}+W_{\mathrm{NL}}[\ell],
$$

where $A_{\alpha \beta}$ and $V_{\beta}$ are the interfacial area and the volume of the $\beta$ layer, respectively. The binding potential functional has the diagrammatic representation

$$
W_{\mathrm{NL}}[\ell]=a_{1} \widetilde{\mathcal{C}}+b_{1} \widehat{\boldsymbol{D}}+\cdots
$$

with geometry independent coefficients $a_{1}=2 t \sigma_{\alpha \beta}, b_{1}=$ $\sigma_{\alpha \beta}$. The structure of $W_{\mathrm{NL}}$ is largely unchanged beyond

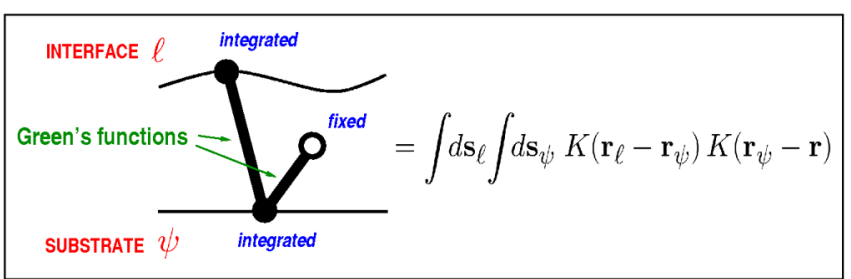

FIG. 1 (color online). Wetting diagram and its algebraic expression. 
DP and when coupling to a surface field is allowed, although the values of $a_{1}$ and $b_{1}$ are slightly altered [6]. When the interface and wall are planar, $W_{\mathrm{NL}}[\ell]=$ $A_{\alpha \beta} W(\ell)$, where $W(\ell)=a_{1} e^{-\kappa \ell}+b_{1} e^{-2 \kappa \ell}$ is the usual binding potential function $[1,11]$. For more general interfacial configurations, the diagrams further simplify to

$$
\begin{gathered}
\mathcal{C}=\int d \mathbf{s} e^{-\kappa \ell} \\
\boldsymbol{\nabla}=\iint d \mathbf{s}_{1} d \mathbf{s}_{2} e^{-2 \kappa \bar{\ell}} S\left(x_{12} ; \bar{\ell}\right)
\end{gathered}
$$

where $\bar{\ell} \equiv\left[\ell\left(\mathbf{x}_{1}\right)+\ell\left(\mathbf{x}_{1}\right)\right] / 2$ and $d \mathbf{s}$ is the appropriate infinitesimal area element at the interface. Here

$$
S(x ; \ell) \approx \frac{1}{4 \pi \xi_{\mathrm{NL}}^{2}} e^{-x^{2} / 4 \xi_{\mathrm{NL}}^{2}}
$$

is an effective two-body interfacial interaction whose range is the new length scale $\xi_{\mathrm{NL}}$ discussed earlier. It is natural to interpret $\xi_{\mathrm{NL}} \propto \ell^{1 / 2}$ as the rms width arising from the thermal wandering of a tube of length $\ell$.

The MF expression for $G^{\text {sing }}$ can be recovered from the interfacial correlation function $\left\langle\delta \ell\left(\mathbf{x}_{1}\right) \delta \ell\left(\mathbf{x}_{2}\right)\right\rangle$, where $\delta \ell \equiv \ell-\langle\ell\rangle$. Using the constrained profile (8) to reconstruct magnetization correlations, we are led to a diagrammatic formula for the correlation function $G$ between two points (open circles),

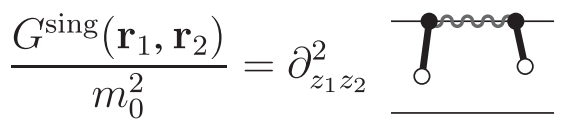

where the horizontal lines denote the equilibrium MF positions of the interface and wall, while the thick line and black dots are as before. The wiggly line represents $\left\langle\delta \ell\left(\mathbf{x}_{1}\right) \delta \ell\left(\mathbf{x}_{2}\right)\right\rangle$ and, therefore, shows magnetization correlations arising from the interaction between tubes and capillary waves. The parallel FT of Eq. (14) leads to the same expression for $G^{\text {sing }}$ obtained in the Landau theory, Eq. (4).

We are now in a position to reexamine some longstanding problems of wetting theory. For complete wetting, the correlation function near the wall is

$$
\mathcal{G}^{\operatorname{sing}}(0,0 ; q) \propto \frac{h^{2}}{2 m_{0} \kappa|h|+\sigma_{\alpha \beta} q^{2}} e^{-q^{2} \xi_{\mathrm{NL}}^{2}},
$$

which remains valid beyond MF. Expanding $G(0,0 ; q)=$ $G_{0}(0,0)-q^{2} G_{2}(0,0)+\ldots$, we find for the second moment

$$
G_{2}^{\text {sing }}(0,0) \propto \sigma_{\alpha \beta}+2 m_{0}|h| \xi_{\mathrm{NL}}^{2} .
$$

This precisely satisfies Henderson's exact sum rule $G_{2}^{\text {sing }}(0,0) \propto \sigma_{\alpha \beta}+\sigma_{\text {sing }}$ [8], identifying correctly the sin- gular contribution to the wall- $\alpha$ surface tension $\sigma^{\text {sing }} \sim$ $h \ln |h|$. The latter term in $G_{2}$ is not captured by the standard local interfacial model since it does not include the exponential damping appearing in the numerator of (15) [14].

We now turn to critical wetting. The crucial observation here is that $\xi_{\mathrm{NL}}$ serves to cut off the spectrum of interfacial interactions that control the repulsion from the wall. This can already be seen in the LGW model results (6) and (7) for the MF correlation function. For example, near the interface and for wave vectors in the range $1 / \xi_{\mathrm{NL}} \lesssim q \lesssim$ $\kappa$, we find $G(\ell, \ell ; q)=\kappa^{2} m_{0}^{2} / \sigma_{\alpha \beta} q^{2}$, which is the same as for a free $\alpha \beta$ interface, independent of the presence of the wall. Similarly, near the wall, the exponential damping kills the singular contribution for $q \gtrsim 1 / \xi_{\mathrm{NL}}$. These effects can be traced to the two-body interaction (13) controlling the repulsion. The FT of the interaction is $\tilde{S}(q) \approx$ $\exp \left(-q^{2} \xi_{\mathrm{NL}}^{2}\right)$, which suppresses strongly the repulsion for wave vectors $q \gtrsim 1 / \xi_{\mathrm{NL}}$. We are now in a position to critically reassess previous predictions for fluctuation effects [1,7]: (a) If we take the ultralong wavelength approximation $\tilde{S}(q) \approx 1$, Eq. (12) reduces to the local interaction $\int d \mathbf{x} e^{-2 \kappa \ell}$, appearing in the original interfacial theory [1]. However, this approximation is only valid provided we restrict wave vectors to $q<\Lambda_{\mathrm{NL}}$ where $\Lambda_{\mathrm{NL}} \sim 1 / \xi_{\mathrm{NL}}$ is an effective momentum cutoff. This contrasts with the original assumption [1] that $q<\Lambda$, where $\Lambda \sim \kappa$ is the high-momentum cutoff [which remains valid for the attractive term (11)]. This implies that the renormalized repulsion should be [13]

$$
R\left[e^{-2 \kappa \ell}\right] \approx \frac{1}{\sqrt{2 \pi} w_{\perp}} \int_{0}^{\infty} d t e^{-2 \kappa t-(\ell-t)^{2} / 2 w_{\perp}^{2}},
$$

determined by a reduced width $\kappa^{2} w_{\perp}^{2}=\omega \ln \left(1+\xi_{\|}^{2} \Lambda_{\mathrm{NL}}^{2}\right)$. Equivalently, the wetting parameter controlling the renormalized repulsion takes a lower effective value

$$
\frac{\omega_{\text {eff }}}{\omega} \approx \frac{\ln \left(1+\xi_{\|}^{2} \Lambda_{\mathrm{NL}}^{2}\right)}{\ln \left(1+\xi_{\|}^{2} \Lambda^{2}\right)},
$$

which, for thick wetting films, reduces to

$$
\omega_{\text {eff }}=\omega-\sqrt{2 \omega^{3}} \frac{\ln (\kappa \ell)}{\kappa \ell}+\cdots,
$$

where we have assumed that $\omega>1 / 2$ as appropriate to the Ising model. (b) The same mechanism prevents the possibility of a stiffness instability subsequently predicted for models that allow for a position-dependent stiffness [7]. Indeed, the Fisher-Jin model can be recovered explicitly from the NL theory if we approximate $\tilde{S}(q) \approx 1-q^{2} \xi_{\mathrm{NL}}^{2}$ [5]. Again, this is valid only for $q<\Lambda_{\mathrm{NL}} \sim 1 / \xi_{\mathrm{NL}}$ and with this corrected cutoff the flow equations studied in [7] no longer drive the transition first order. Importantly, the NL theory preserves the Nakanishi-Fisher global phase diagram showing first order, critical, and tricritical wetting 


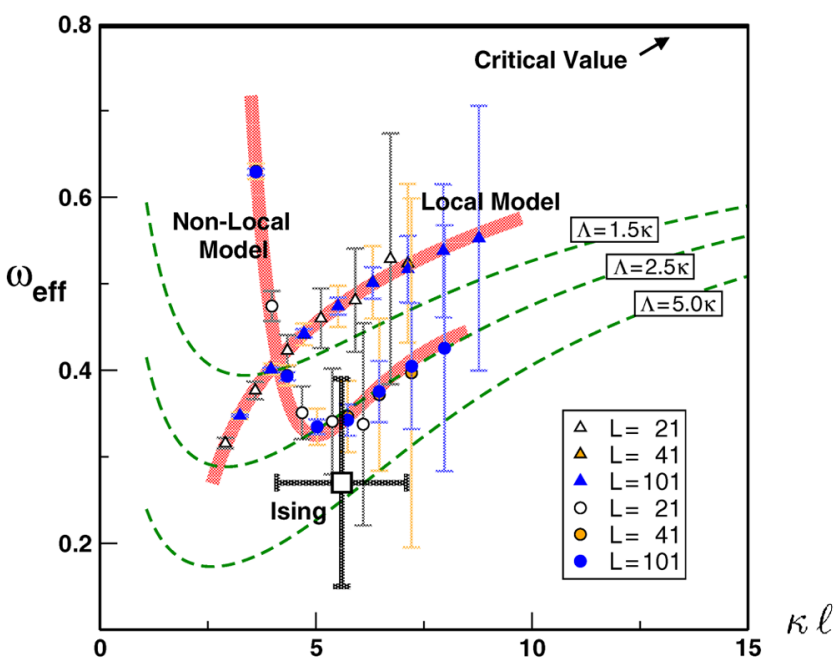

FIG. 2 (color online). Effective value of the wetting parameter as a function of the wetting thickness. Simulations of the discretized local (triangles) and nonlocal (circles) interfacial models were performed on an $L \times L$ grid. Here $L$ is measured in units of $3.1623 / \kappa$ [4]. The thick lines are guides for the eye. The continuous and dashed lines are predictions of the continuum approximation (18) for different $\Lambda$. The value of $\omega_{\text {eff }}$ obtained from Ising model simulations [3] is also shown (square).

[10]. All these considerations are consistent with the more detailed analysis based on the numerical renormalization of the two-body interaction (13) given in [4].

It is this reduced value $\omega_{\text {eff }}$ that determines the singularities of the first-layer magnetization and susceptibility along the critical wetting isotherm, which were studied in simulations of the Ising model [2] and NL Hamiltonian [4]. In Fig. 2, we plot $\omega_{\text {eff }}$ vs the film thickness $\kappa \ell$ as obtained from simulation studies and the above theory. In the simulation studies of the interfacial models, $\omega_{\text {eff }}$ has been extracted from the singularity of the surface magnetization $m_{1} \sim|h|^{1-1 / 2 \nu_{\|}\left(\omega_{\text {eff }}\right)}$ along the critical wetting isotherm. This is more difficult for the Ising model, and we have used the estimate taken from the surface susceptibility critical amplitude [3]. As can be seen, the NL theory is in better agreement with the Ising model simulation result due to the slower crossover. Theoretical predictions for $\omega_{\text {eff }}$ are also shown for different values of $\Lambda$. This is close to the simulation findings and substantially lower than the asymptotic critical value $\omega=0.8$. It is interesting to note that, within the NL model, $\omega_{\text {eff }}$ has a minimum value of approximately 0.3 when the film thickness is approximately 5 bulk correlation lengths. For thinner wetting films, the effective value actually increases. Similar qualitative behavior is found in Eq. (18). Unfortunately, in the original Ising simulation studies, this regime corresponds to wetting layers less than 1 or 2 lattice spacings for which a continuum description is doubtful. However, this limitation can be overcome in future simulations at temperatures closer to $T_{c}$, where the bulk correlation length is much larger.

In summary, we have shown that 3D short-ranged wetting transitions are characterized by two diverging parallel length scales. The existence of a new length $\xi_{\mathrm{NL}}$ appears to resolve long-standing puzzles in wetting theory. In particular, for critical wetting it suppresses long-wavelength interfacial modes, implying that critical singularities are necessarily much closer to mean-field predictions in keeping with Ising model simulation results.

C. R., N. R. B., and J. M. R-E. acknowledge support from grants MOSAICO and MOSSNOHO, the Portuguese FCT and Grant No. ENE2007-6804-C03.

[1] E. Brézin, B. I. Halperin, and S. Leibler, Phys. Rev. Lett. 50, 1387 (1983); D. S. Fisher and D. A. Huse, Phys. Rev. B 32, 247 (1985).

[2] K. Binder, D. P. Landau, and D. M. Kroll, Phys. Rev. Lett. 56, 2272 (1986); K. Binder, D. P. Landau, and S. Wansleben, Phys. Rev. B 40, 6971 (1989).

[3] A. O. Parry, R. Evans, and K. Binder, Phys. Rev. B 43, 11535 (1991).

[4] A. O. Parry, J.M. Romero-Enrique, and A. Lazarides, Phys. Rev. Lett. 93, 086104 (2004).

[5] A. O. Parry, C. Rascón, N. Bernardino, and J. M. RomeroEnrique, J. Phys. Condens. Matter 18, 6433 (2006).

[6] A. O. Parry, C. Rascón, N. Bernardino, and J. M. RomeroEnrique, J. Phys. Condens. Matter 19, 416105 (2007).

[7] M. E. Fisher and A. J. Jin, Phys. Rev. Lett. 69, 792 (1992); A. J. Jin and M.E. Fisher, Phys. Rev. B 48, 2642 (1993).

[8] J.R. Henderson, in Fundamentals of Inhomogeneous Fluids, edited by D. Henderson (Dekker, New York, 1992), Ch. 2.

[9] J. W. Cahn, J. Chem. Phys. 66, 3667 (1977); C. Ebner and W. F. Saam, Phys. Rev. Lett. 38, 1486 (1977).

[10] H. Nakanishi and M.E. Fisher, Phys. Rev. Lett. 49, 1565 (1982).

[11] S. Dietrich, in Phase Transitions and Critical Phenomena, edited by C. Domb and J.L. Lebowitz (Academic, New York, 1988), vol. 12.

[12] R. Evans, D. C. Hoyle, and A. O. Parry, Phys. Rev. A 45, 3823 (1992); M. E. Fisher and H. Wen, Phys. Rev. Lett. 68, 3654 (1992).

[13] A. O. Parry, C. Rascón, N. Bernardino, and J. M. RomeroEnrique (to be published).

[14] A. O. Parry and R. Evans, Mol. Phys. 78, 1527 (1993); L. V. Mikheev and J.D. Weeks, Physica (Amsterdam) 177A, 495 (1991). 